Transactions of the 13th International Conference on Structural Mechanics in Reactor Technology (SMiRT 13), Escola de Engenharia - Universidade Federal do Rio Grande do Sul, Porto Alegre, Brazil, August 13-18, 1995

\title{
Modified solution of radial nozzle with thick reinforcement in spherical vessel head subjected to radial load
}

Lee, Y.S.,

Chungnam National University, Dept. of Mechanical Design Engineering, Taejon, Korea

Sohn, Y.-S.

Korea Atomic Research Institute, Dept. of Mechanical Engineering, Taejon, Korea

\begin{abstract}
When nozzles in the spherical vessel head are designed by area replacement method defined in the majority of pressure vessel code, the thickness of the reinforcements is so thick that the application of the thin shell theory may not be appropriate in the shell intersection problems. To obtain the solution of the thick reinforcement in the radial nozzle of the spherical vessel head subjected to radial load, the nature of thick shell is introduced to the solution of thin shell: (1) modified meridional moment at the junction should be used in moment equilibrium equation instead of meridional moment at mid-plane of reinforcement, (2) angular rotation derivative may be neglected in the meridional moment equation, or (3) double derivative of radial displacement may be neglected in meridional moment equation. These modified solutions are found to be reasonable and be able to use for the design of nozzle with the thick reinforcement in the initial design stage.
\end{abstract}

\section{INTRODUCTION}

Openings in pressure vessels are usually inevitable and constitute a major source of weakness. The majority of pressure vessel codes including ASME (1992) use the principle of "area replacement" for the design of nozzles and it is well-known that this simple principle leads to design for which the elastic stress concentration factor and limit pressure can vary widely.

One of the typical thin shell structures used in pressure vessel design is that of a spherical vessel head with a cylindrical vessel and some radial nozzles in the vessel head. Such a pressure vessel is commonly used in many industries, including nuclear power plant.

In addition, some reinforcement structures are often adopted by the pressure vessel design for a spherical head with radial nozzles. When welded to a shell plate, a branch may or may not protrude through the shell depending on general design requirements as well as those of strength. If reinforcement around the opening is required, it can be provided in a number of different ways as shown in Figs. 1, 2 and 3: (1) increasing thickness of the nozzle (nozzle integral reinforcement), (2) increasing the thickness of the main shell in the vicinity of the opening (shell integral reinforcement), or (3) welding a pad to the main shell (pad reinforcement).

The solutions for nozzles in spherical shells were presented by Bijlaard and Leckie in 1959 and 1961, respectively. Bijlard's work is based on the asymptotic integration of the differential equations for the thin shallow spherical shell and Leckie's work based on the differential equations for the thin spherical shell. In both analyses, as is usual with shell assembly problems the shells are assumed to join at the intersections of the median lines of 
their thickness at the junctions as shown Figs. 1(b), 2(b) and 3(b), which are the junction of cylinder and sphere (reinforcement) and the junction of two spheres with different thickness (reinforcement and main shell).

Limit analysis of radial nozzles in spherical shells was reported by Cloud in 1965. In this analysis the reinforcement was considered as a solid ring (rigid body) instead of a part of shell.

As recent works, the theoretical analysis expanded the Leckie solution to the pad reinforced radial nozzle (Fig. 3(a)) in spherical vessel heads were reported by Gill et. al in 1978 and theoretical analysis for the reinforced radial nozzles in ellipsoidal vessel head was reported by Chang in 1994, which expands the Leckie theory to the ellipsoidal shell.

In nuclear power plant the number of piping supports tends to be reduced or all piping supports be removed in piping design due to the application of leak before break concept. As the results, external nozzle loads due to piping behavior are highly increased and the thickness of the reinforcement increases. Even though the increased thickness of the reinforcement is still within the range of the thin shell that the ratio of the thickness to radius is less than 0.1 , the middle planes of reinforcement and the main shell may not coincide as shown Figs. 1(a) and 2(a). It is unreasonable to apply Bijlaard's or Leckie's solution in this problem without any correction of equilibrium equation at the junction of reinforcement and main shell since the effect due to the difference of distance, $\delta_{t}$ (Fig. 4), between middle planes of reinforcement and main shell is not negligible as the thickness of the reinforcement increases. The appropriate analysis is required to resolve this problem in the initial design stage.

To resolve this problem, the effect due to the difference of distance between middle planes of reinforcement and main shell shall be considered in the calculation of moments of the reinforcement at the junction of the reinforcement and main shell, $\phi_{2}$.

In high pressure vessel the thickness of the reinforcement determined by area replacement method is generally thick and the ratio of the reinforcement thickness to the radius is beyond the range of the thin shell. Bijlaard or Leckie's thin shell theories may not produce reasonable results for these cases. The deformation of the thick reinforcement may show the characteristics of rigid body as the thickness increases. The ring reinforcement method by Cloud (1965) has commonly been used for the thick reinforcement in the initial nozzle design stage. However the ring reinforcement method is not appropriate to obtain the stresses in the ring reinforcement since ring reinforcement is dealt with a solid plug (rigid body) in the method. It is also much difficult to find the solutions for the thick shell.

To resolve this problem for the thick reinforcement, a modified theory can be introduced by considering the nature of thick shell to the solutions of thin shell: (1) angular rotation derivative with respect to $\phi, \dot{\beta}$, are neglected in the moment equations, or (2) double derivative of radial displacement with respect to $\phi, \partial^{2} w / \partial \phi^{2}$, are neglected in the moment equations.

\section{THEORETICAL ANALYSIS}

The structure being analyzed is shown in Fig. 4 where the geometric parameters are defined. It consists for the purposes of analysis of a spherical vessel (main shell), integral reinforcement (referred to as reinforcement), and a semi-infinite cylindrical shell (the flush branch connected to the spherical vessel).

In the analysis of the structure under the action of a radial load applied to the branch, the geometric assumptions are as follows: (1) intersection point of sphere and cylinder is the intersection of the middle plane of reinforcement and middle plane of cylinder, (2) intersection point of reinforcement and main shell is the normal plane of reinforcement and 
the middle plane of main shell at $\phi_{2}$ (Fig. 4).

For each part of the structure, the complete solution is found by superimposing an edge solution on a membrane solution. The solutions for cylinder are given by Kraus (1967). For the sphere the solutions given by Leckie (1961) are used with some modification according to the each analysis of case. In both solutions there is no limitations on the geometrical parameters.

According to the different analyses for the thick reinforcement, the detail analysis is distinguished by the four cases.

Case 1: No correction for geometry and no modification of solution (referred to as current method); even though the middle planes of the reinforcement and the main shell are not coincide and the thickness of the reinforcement is beyond the range of the thin shell, it is assumed that their middle planes are coincide and the reinforcement is the thin shell.

Case 2: Correction for geometry and no modification of solution(referred to as moment modified method); even though the thickness of the reinforcement is beyond the range of the thin shell, it is assumed that the reinforcement is the thin shell. Only the effect due to the difference of distance, $\delta_{t}$ (Fig. 4), between middle planes of reinforcement and main shell is considered in the calculation of moments of the reinforcement at junction of the reinforcement and main shell, $\phi_{2}$.

Case 3: Correction for geometry and modification of rotation function in solution (referred to as rotation modified method); angular rotation derivative, $\dot{\beta}$, is neglected in the thin shell solution which means that the rotation of the thick reinforcement may be constant through all reinforcement section, to reflect the characteristics of the thick shell on the solution of the thin shell and the effect due to the difference of distance between middle planes of reinforcement and main shell is considered the same as Case 2.

Case 4: Correction for geometry and modification of radial displacement in solution (referred to as displacement modified method); double derivative of radial displacement, $\partial^{2} w / \partial \phi^{2}$, is neglected in the thin shell solution which means that the thick reinforcement may be deformed radially as a linear displacement function through the reinforcement section, to reflect an other characteristics of the thick shell on the solution of thin shell and the effect due to the difference of distance between middle planes of reinforcement and main shell is considered the same as Case 2.

Figure 5 shows membrane and edge forces and moments acting on the junctions of the components of the structure. Note that the axial components of the membrane forces $P_{2}, P_{3}$, and $\mathrm{P}_{4}$ will be accompanied for a membrane solution, by horizontal components $\mathrm{H}_{\mathrm{m}}, \mathrm{H}_{\mathrm{m} 2}$, and $\mathrm{H}_{\mathrm{m} 3}$ where:

$(1 \mathrm{a}, \mathrm{b}, \mathrm{c}) \quad \mathrm{H}_{\mathrm{m} 2}=\mathrm{P}_{2} \cot \phi_{1}, \quad \mathrm{H}_{\mathrm{m} 3}=\mathrm{P}_{3} \cot \phi_{2}, \quad \mathrm{H}_{\mathrm{m} 4}=\mathrm{P}_{4} \cot \phi_{2}$

The general expression used in the analysis for each of the components parts of the structure are given below.

\section{Cylinder}

At a distance $x$ from the edge of the cylinder (see Fig. 4) the stress resultants due to membrane and edge forces as follows, the shell being assumed semi-infinite (Kraus, 1967):

$(6 a, b)$ where,

$$
\begin{aligned}
& N_{x}=\frac{P}{2 \pi r_{c}} \\
& N_{\theta}=-\frac{e^{-\mu_{c} x} E t_{c}}{2 \mu_{c}^{3} D_{c} r_{c}}\left[H_{1} \cos \mu_{c} x-\mu_{c} M_{1}\left(\cos \mu_{c} x-\sin \mu_{c} x\right)\right] \\
& M_{x}=-\frac{e^{-\mu_{c} x}}{\mu_{c}}\left[H_{1} \sin \mu_{c} x-\mu_{c} M_{1}\left(\cos \mu_{c} x+\sin \mu_{c} x\right)\right] \\
& M_{\theta}=v M_{x} \\
& \mu_{c}^{4}=\frac{3\left(1-v^{2}\right)}{r_{c}^{2} t_{c}^{2}}, \quad D_{c}=\frac{E_{c}^{3}}{12\left(1-v^{2}\right)}
\end{aligned}
$$


The deformation of radius and rotation of a generator are:

$$
\begin{aligned}
& w=\delta_{h}=-\frac{e^{-\mu_{c} x}}{2 \mu_{c}^{3} D_{c}}\left[H_{1} \cos \mu_{c} x-\mu_{c} M_{1}\left(\sin \mu_{c} x-\cos \mu_{c} x\right)\right]-\frac{v}{2 \pi E t_{c}} P \\
& \beta=-\frac{e^{-\mu_{c} x}}{2 \mu_{c}^{2} D_{c}}\left[H_{l}\left(\cos \mu_{c} x+\sin \mu_{c} x\right)+2 \mu_{c} M_{1} \cos \mu_{c} x\right]
\end{aligned}
$$

The values of $\delta_{h}$ and $\beta$ at juncture 1 where $x=0$ are $\delta_{h 1}$ and $\beta_{1}$ and they may be written in terms of $\mathrm{H}_{1}$ and $\mathrm{M}_{1}$.

\section{Reinforcement}

This has two boundaries and is subjected to external load on the inner and outer edges. The stress resultants due to membrane and edge forces at any meridional position defined by $\phi$ are given by Leckie (1961):

$$
\begin{aligned}
& \mathrm{Q}_{\phi}=\frac{\mathrm{K}_{\mathrm{p}}}{\mathrm{R}_{\mathrm{p}}}\left(\mathrm{A}_{1} \mathrm{~T}_{1}+\mathrm{A}_{2} \mathrm{~T}_{2}+\mathrm{A}_{3} \mathrm{~T}_{3}+\mathrm{A}_{4} \mathrm{~T}_{4}\right) \\
& \mathrm{N}_{\phi}=-\mathrm{Q}_{\phi} \cot \phi+\mathrm{P}_{2} \frac{\sin \phi_{1}}{\sin ^{2} \phi} \\
& N_{\theta}=-\frac{K_{p}}{R_{p}}\left(A_{1} \dot{T}_{1}+A_{2} \dot{T}_{2}+A_{3} \dot{T}_{3}+A_{4} \dot{T}_{4}\right)-P_{2} \frac{\sin \phi_{1}}{\sin ^{2} \phi} \\
& M_{\phi}=\frac{D_{p}}{R_{p}}(\dot{\beta}+v \beta \cot \phi)=\frac{D_{p}}{R_{p}}\left[\left(\frac{\partial^{2} w}{\partial \phi^{2}}-\frac{\partial v}{\partial \phi}\right)+v \beta \cot \phi\right] \\
& =\frac{2 \kappa_{p}^{2} D_{p}}{R_{p}^{2}\left(1-v^{2}\right)}\left\{A_{1}\left[\dot{T}_{2}+v T_{2} \cot \phi-\frac{v}{2 \kappa_{p}^{2}}\left(\dot{T}_{1}+v T_{1} \cot \phi\right)\right]\right. \\
& -A_{2}\left[\dot{T}_{1}+v T_{1} \cot \phi+\frac{v}{2 \kappa_{p}^{2}}\left(\dot{T}_{2}+v T_{2} \cot \phi\right)\right] \\
& +A_{3}\left[\dot{\mathrm{T}}_{4}+v \mathrm{~T}_{4} \cot \phi-\frac{v}{2 \kappa_{\mathrm{p}}^{2}}\left(\dot{\mathrm{T}}_{3}+v \mathrm{~T}_{3} \cot \phi\right)\right] \\
& \left.-\mathrm{A}_{4}\left[\dot{\mathrm{T}}_{3}+v \mathrm{~T}_{3} \cot \phi+\frac{v}{2 \kappa_{\mathrm{p}}^{2}}\left(\dot{\mathrm{T}}_{4}+v \mathrm{~T}_{4} \cot \phi\right)\right]\right\} \\
& M_{\theta}=\frac{D_{p}}{R_{p}}(v \dot{\beta}+\beta \cot \phi)=\frac{D_{p}}{R_{p}}\left[v\left(\frac{\partial^{2} w}{\partial \phi^{2}}-\frac{\partial v}{\partial \phi}\right)+\beta \cot \phi\right] \\
& =\frac{2 \kappa_{p}^{2} D_{p}}{R_{p}^{2}\left(1-v^{2}\right)}\left\{A_{1}\left[v \dot{T}_{2}+T_{2} \cot \phi-\frac{v}{2 \kappa_{p}^{2}}\left(v \dot{T}_{1}+T_{1} \cot \phi\right)\right]\right. \\
& -\mathrm{A}_{2}\left[v \dot{\mathrm{T}}_{1}+\mathrm{T}_{1} \cot \phi+\frac{v}{2 \kappa_{\mathrm{p}}^{2}}\left(v \dot{\mathrm{T}}_{2}+\mathrm{T}_{2} \cot \phi\right)\right] \\
& +A_{3}\left[v \dot{T}_{4}+T_{4} \cot \phi-\frac{v}{2 \kappa_{p}^{2}}\left(v \dot{T}_{3}+T_{3} \cot \phi\right)\right] \\
& \left.-\mathrm{A}_{4}\left[v \dot{\mathrm{T}}_{3}+\mathrm{T}_{3} \cot \phi+\frac{v}{2 \kappa_{\mathrm{p}}^{2}}\left(v \dot{\mathrm{T}}_{4}+\mathrm{T}_{4} \cot \phi\right)\right]\right\}
\end{aligned}
$$

(14a,b,c) where,

$$
K_{p}=\frac{E t_{p}}{\left(1-v^{2}\right)}, \quad D_{p}=\frac{E t_{p}^{3}}{12\left(1-v^{2}\right)}, \quad \kappa_{p}^{4}=\frac{1}{\sqrt{2}}\left[\frac{12\left(1-v^{2}\right) R_{p}^{2}}{t_{p}^{2}}-v^{2}\right]
$$

The deformation of radius of the parallel circle is:

$$
\delta_{h}=\frac{R_{p} \sin \phi}{E t_{p}}\left(N_{\theta}-v N_{\phi}\right)
$$

The angular rotation of a tangent to a meridian is: 


$$
\begin{aligned}
\beta & =\frac{1}{R_{p}}\left(\frac{\partial w}{\partial \phi}-v\right) \\
& =\frac{2 \kappa_{p}^{2}}{R_{p}\left(1-v^{2}\right)}\left\{A_{1}\left[T_{2}-\frac{v}{2 \kappa_{p}^{2}} T_{1}\right]-A_{2}\left[T_{1}-\frac{v}{2 \kappa_{p}^{2}} T_{2}\right]+A_{3}\left[T_{4}-\frac{v}{2 \kappa_{p}^{2}} T_{3}\right]-A_{4}\left[T_{3}-\frac{v}{2 \kappa_{p}^{2}} T_{4}\right]\right\}
\end{aligned}
$$

Where $A_{1}, A_{2}, A_{3}, A_{4}$ are arbitrary constants and the functions $T_{1}$ are:

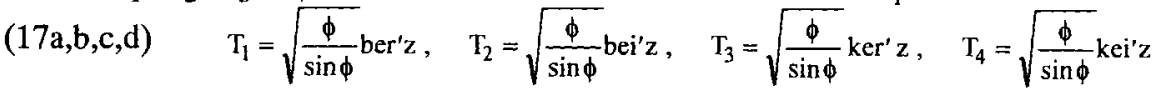

(18) where,

$$
z=\sqrt{2} \kappa_{p} \phi
$$

\section{Juncture 1}

Using equation (9);

$$
\mathrm{H}_{2}=\mathrm{Q}_{\phi_{1}} / \sin \phi_{1}
$$

Equations (12), (15) and (16) give $\mathrm{M}_{2}, \delta_{\mathrm{h} 2}$, and $\beta_{2}$ directly. Suffices $1,2,3$ and 4 refer to the appropriate edge defined in Fig. 5.

Juncture 2

In thick shell the rotation can be assumed to be constant or the rotation derivative be very small and negligible along the meridional direction. In the analysis of case 3 , the rotation derivative, $\dot{\beta}$, is neglected from the equations (12) and (13). Another assumption in thick shell is that the radial displacement can be assumed to be changed linearly and the double derivative of radial displacement be negligible. In analysis of case 4 , the double derivative of radial displacement, $\partial^{2} w / \partial \phi^{2}$, is neglected from the equations (12) and (13).

All these equations are expressed in terms of the unknown constants $A_{1}, A_{2}, A_{3}, A_{4}$ and external load $\mathbf{P}$ for a given geometry and material. Using the same equations and substituting $\phi_{2}$ for $\phi$, similar expressions may be determined for $\mathrm{H}_{3}, \mathrm{M}_{3}, \delta_{\mathrm{h} 3}, \beta_{3}$ and in terms of $\mathrm{A}_{1}, \mathrm{~A}_{2}, \mathrm{~A}_{3}, \mathrm{~A}_{4}$ and external load $\mathrm{P}$

Main Shell

Expressions for $\mathrm{H}_{4}, \mathrm{M}_{4}, \delta_{\mathrm{h} 4}, \beta_{4}$ are calculated as for $\mathrm{H}_{2}, \mathrm{M}_{2}, \delta_{\mathrm{h} 2}, \beta_{2}$ by replacing by $\mathrm{R}_{\mathrm{p}}$ by $R_{s}, t_{p}$ by $t_{s}$ and $P_{2}$ by $P_{4}$ with the constants $A_{1}, A_{2}, A_{3}, A_{4}$ by $A_{1}^{\prime}, A_{2}^{\prime}, A_{3}^{\prime}, A_{4}^{\prime}$. Since this shell has only one boundary:

$$
A_{1}^{\prime}=A_{2}^{\prime}=0
$$

Unknown in these expressions are now $A_{3}^{\prime}, A_{4}^{\prime}$, and external load $P$.

\section{Compatibility Equations}

It is assumed that there is no relative displacement of rotation between the component parts of the structure at each junction. At juncture 1, the junction between cylinder and reinforcement, it follows that:

$$
\begin{aligned}
& \delta_{\mathrm{h} 1}=\delta_{\mathrm{h} 2} \\
& \beta_{\mathrm{hl}}=\beta_{\mathrm{h} 2}
\end{aligned}
$$

Similarly, at juncture 2, the junction between reinforcement and main shell, it follows that:

$$
\begin{aligned}
& \delta_{h 4}=\delta_{h 3} \\
& \beta_{h 4}=\beta_{h 3}
\end{aligned}
$$

\section{Equilibrium Equations}

At juncture 1, horizontal force equilibrium is:

$$
\mathrm{H}_{1}-\mathrm{H}_{2}=-\frac{P \cot \phi_{1}}{2 \pi r_{c}}
$$

Moment equilibrium is:

$$
\mathrm{M}_{1}-\mathrm{M}_{2}=0
$$

At juncture 2, horizontal force equilibrium is:

$$
\mathrm{H}_{4}-\mathrm{H}_{3}=0
$$


Moment equilibrium is:

(28)

$$
\mathrm{M}_{4}-\mathrm{CF} \cdot \mathrm{M}_{3}=0
$$

Where $\mathrm{CF}$ is correction factor due to difference distance between reinforcement middle plane and main shell middle plane. For the analysis of case 1 the value is one and for the other analysis of cases the values are calculated as follows:

$$
\mathrm{CF}=3 \frac{\mathrm{t}_{\mathrm{s}}}{\mathrm{t}_{\mathrm{p}}}-3\left[\frac{\mathrm{t}_{\mathrm{s}}}{\mathrm{t}_{\mathrm{p}}}\right]^{2}+\left[\frac{\mathrm{t}_{\mathrm{s}}}{\mathrm{t}_{\mathrm{p}}}\right]^{3}
$$

\section{Solution}

By the use of equation (2) to (8) for the cylinder equations, (9) to (19) for the reinforcement, with similar expressions for the main shell, the eight equations (21) to (28), may be set up in terms of the eight unknowns $\mathrm{H}_{1}, \mathrm{M}_{1}, \mathrm{~A}_{1}, \mathrm{~A}_{2}, \mathrm{~A}_{3}, \mathrm{~A}_{4}, \mathrm{~A}_{3}^{\prime}$ and $\mathrm{A}_{4}^{\prime}$ and external load, $P$, in matrix form .

The solution for a given thrust load $\mathrm{P}$ was obtained by means of a computer analysis.

\section{Stresses}

Stress distributions were calculated using equations (2) to (8) for the cylinder, (5) to (13) for the reinforcement and similar expressions for main shell.

\section{RESULTS OF ANALYSIS}

The proceeding theoretical analyses have been employed to calculate the stress distributions in the reinforcement for the nine specimens as follows:

$$
\begin{array}{ll}
t_{\mathrm{P}} / \mathrm{R}_{\mathrm{S}}=0.06, & \mathrm{t}_{\mathrm{P}} / \mathrm{t}_{\mathrm{S}}=1.25 \\
\mathrm{t}_{\mathrm{P}} / \mathrm{R}_{\mathrm{S}}=0.08, & \mathrm{t}_{\mathrm{P}} / \mathrm{t}_{\mathrm{S}}=1.67 \\
\mathrm{t}_{\mathrm{P}} / \mathrm{R}_{\mathrm{S}}=0.10, & \mathrm{t}_{\mathrm{P}} / \mathrm{t}_{\mathrm{S}}=2.08 \\
\mathrm{t}_{\mathrm{P}} / \mathrm{R}_{\mathrm{S}}=0.12, & \mathrm{t}_{\mathrm{P}} / \mathrm{t}_{\mathrm{S}}=2.50 \\
\mathrm{t}_{\mathrm{P}} / \mathrm{R}_{\mathrm{S}}=0.14, & \mathrm{t}_{\mathrm{P}} / \mathrm{t}_{\mathrm{S}}=2.92 \\
\mathrm{t}_{\mathrm{P}} / \mathrm{R}_{\mathrm{S}}=0.15, & \mathrm{t}_{\mathrm{P}} / \mathrm{t}_{\mathrm{S}}=3.33 \\
\mathrm{t}_{\mathrm{P}} / \mathrm{R}_{\mathrm{S}}=0.17, & \mathrm{t}_{\mathrm{P}} / \mathrm{t}_{\mathrm{S}}=3.75 \\
\mathrm{t}_{\mathrm{P}} / \mathrm{R}_{\mathrm{S}}=0.19, & \mathrm{t}_{\mathrm{P}} / \mathrm{t}_{\mathrm{S}}=4.17 \\
\mathrm{t}_{\mathrm{P}} / \mathrm{R}_{\mathrm{S}}=0.20, & \mathrm{t}_{\mathrm{P}} / \mathrm{t}_{\mathrm{S}}=4.58
\end{array}
$$

The geometric parameters, material properties and axial nozzle load used for these analyses are shown in Fig. 4. The required thickness of the reinforcement calculated by area replacement method is 6 inches and the ratio of the thickness to radius is 0.12 . In order to reduce the stresses induced in the reinforcement due to the external piping loads including bending moment, the thickness of reinforcement is increased up to 11 inches (the ratio, 0.2). In order to investigate the effect due to the increase of the reinforcement thickness per the axial nozzle load, even though the reinforcement is beyond the range of the thin shell, Case 1 analysis is carried out to compare with Case 2, Case 3 and 4 analyses.

As a results of calculation, the theoretical stress distributions at the junction of the reinforcement for the nine specimens are shown in four figures for comparison (Fig. 6 to 9).

In case of that the reinforcement is within the range of the thin shell (the ratio less than 0.1 ), the deviation between the moment modified method and the current method must be small since the difference of distance between middle planes of reinforcement and main shell is small. This phenomenon can be found in Fig. 6 through 9. Within the range of thin shell, rotation and displacement modified methods have a big difference from the current method and these two methods seem to be unreasonable within this range.

It is shown beyond the range of thin shell that the deviation between three modified methods and current method is large. When the middle planes are not coincide the deviation is considerably large at the junction of the reinforcement and main shell, $\phi_{2}$. It is found out 
that the current method can not be used in case of that the reinforcement is beyond the range of thick shell and the difference of distance between middle planes of reinforcement and main shell exists.

The deviations between moment modified method and rotation or displacement modified methods exist in the range of thick shell and change with thickness to radius ratio in the range of the thick shell. It can be said that the deviations represent the characteristics of thick reinforcement for the radial load.

The result of the rotation modified method is a little higher than the result of the displacement modified method in the range of the thick shell but the deviation is decreasing with thickness to radius ratio.

In point of conservatism, the rotation and displacement modified methods is better than the moment modified method in the thick shell reinforcement design since the deviation still exists.

\section{CONCLUSIONS}

For three modified methods (Case 2,3 and 4) and current method (Case 1), elastic analyses for nine specimens have been carried out on the stresses in a thick reinforcement of the radial nozzle in a spherical vessel head subjected to a radial load. From the results the characteristics of three modified methods are as follows: The geometric problem of the reinforcement of which the middle planes are not coincide can be resolved by the moment modified method (Case 2).

As the thickness of the reinforcement increases, the current method is not conservative and the rotation and displacement modified methods (Case 3 and 4) can be substituted for current method.

In the initial thick shell reinforcement design for the axial nozzle load, the rotational modified method are recommended for the conservative design.

\section{REFERENCES}

1992. ASME boiler and pressure vessel code, section III for nuclear vessels.

P.P. Bijlaard. 1959. Stress in spherical vessels from radial loads and external moments acting on pipe. Welding Research Council Bulletin. No. 49.

F.A. Leckie. 1961. Localized loads applied to spherical shells. Journal Mechanical Engineering Science. Vol. 3, No. 2:111-118.

R.L. Cloud. 1965.The limit pressure of radial nozzles in spherical shells. Nuclear Structural Engineering. Vol. 1: 403-413.

H. Kraus. 1967. Thin elastic shells. John Wiley \& Sons, Inc.

G. Chang. 1994. Stress analysis of ellipsoidal vessel head with large-diameter flush radial nozzle and comparison of reinforcement types. Int. J. Pres. Ves. \& Piping 60:109-125. 


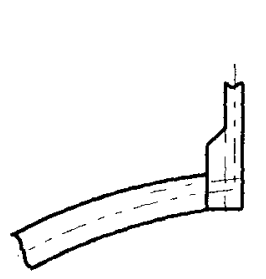

(a) Flush nozzle

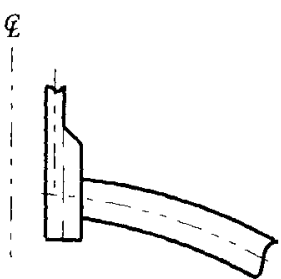

(b) Protruding nozzzle

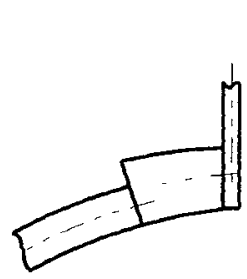

(a) Flush nozzle

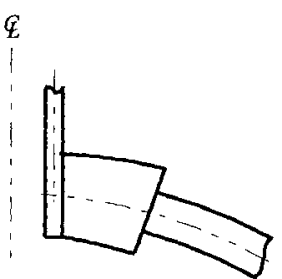

(b) Protruding nozzzle

Fig. 1 Nozzle Integral Reinforcement

Fig. 2 Shell Integral Reinforcement

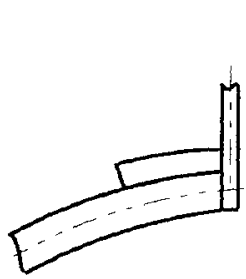

(a) Flush nozzle

(b) Protruding nozzzle

Fig. 3 Pad Reinforcement
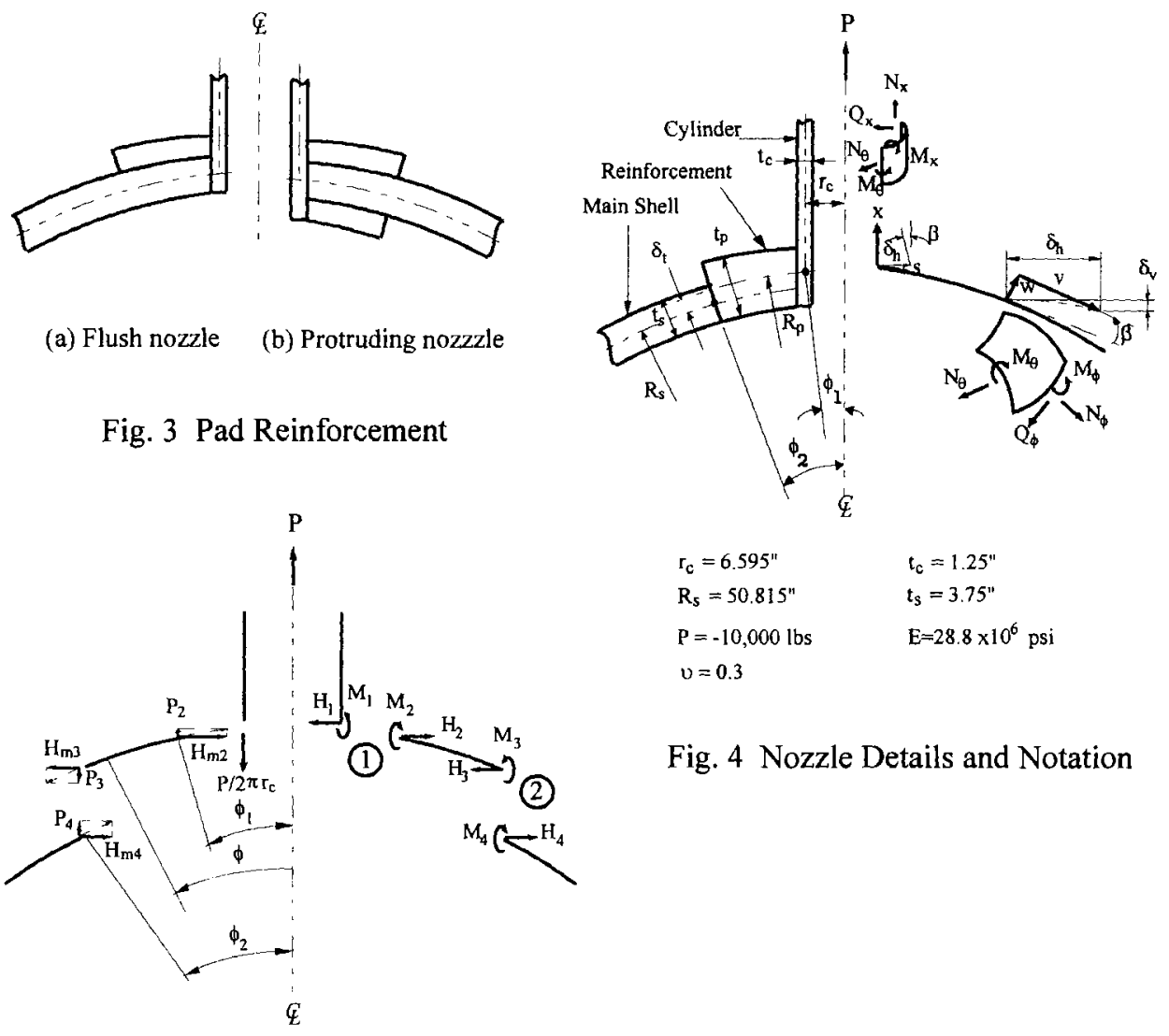

$$
\begin{array}{ll}
\mathbf{r}_{\mathrm{c}}=6.595^{\prime \prime} & \mathrm{t}_{\mathrm{c}}=1.25^{\prime \prime} \\
\mathbf{R}_{\mathbf{S}}=50.815^{\prime \prime} & t_{\mathrm{S}}=3.75^{\prime \prime} \\
\mathbf{P}=-10,000 \mathrm{lbs} & \mathrm{E}=28.8 \times 10^{6} \mathrm{psi} \\
v=0.3 &
\end{array}
$$

Fig. 4 Nozzle Details and Notation
(a) Membrane forces
(b) Edge forces

Fig. 5 Membrane and Edge Forces on Components of Struciure 


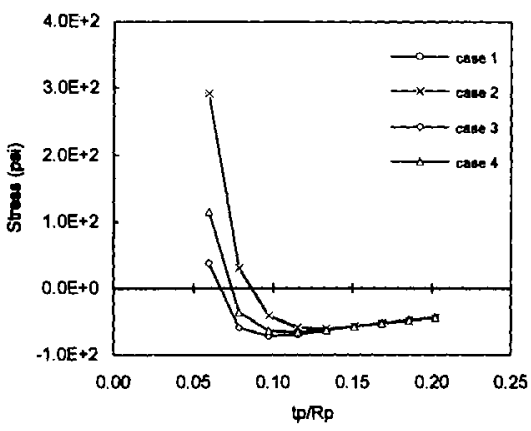

Fig. 6 Meridional Stresses at $\phi_{1}$ on the inside Reinforcement

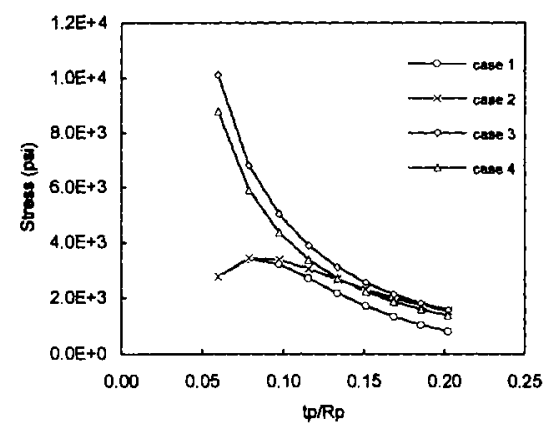

Fig. 8 Circumferential Stresses at $\phi_{1}$ on the Outside Reinforcement

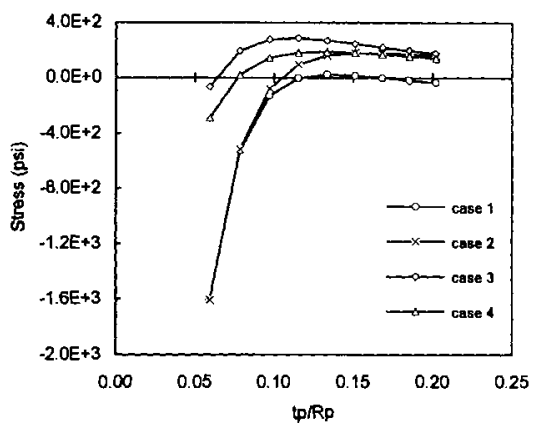

Fig. 7 Meridional Stresses at $\phi_{2}$ on the Inside Reinforcement

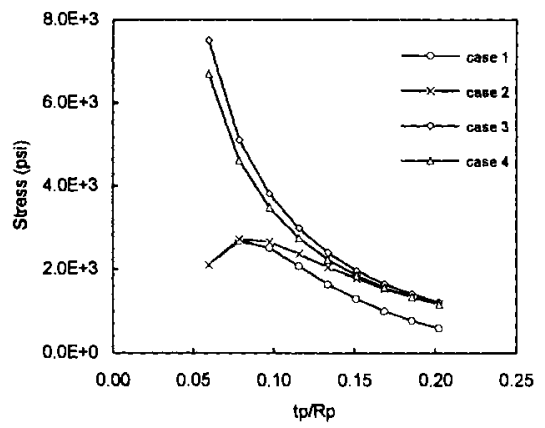

Fig. 9 Circumferential Stresses at $\phi_{2}$ on the Outside Reinforcement 
F06-10 